\title{
Emergent Universe from Many Unreal World Interpretation
}

\author{
Bhushan Bhoja Poojary \\ Bachelor of Science Physics, NIMS University, Jaipur, Rajasthan, India \\ Email address: \\ bhushanpoojary@gmail.com
}

\section{To cite this article:}

Bhushan Bhoja Poojary. Emergent Universe from Many Unreal World Interpretation. International Journal of Applied Mathematics and Theoretical Physics. Vol. 6, No. 3, 2020, pp. 14-18. doi: 10.11648/j.ijamtp.20200602.11

Received: April 11, 2020; Accepted: May 26, 2020; Published: June 15, 2020

\begin{abstract}
There are multiple interpretations of quantum mechanics, 2 most famous interpretations are Copenhagen interpretation and many world interpretations. Copenhagen interpretation fails to explain the mechanism behind wave function collapse and many world interpretations avoid collapse by adding infinite branching worlds. Goal of this paper is to provide alternate interpretations which explains mechanism behind collapse using finite pre-existent unreal worlds. All quantum weirdness can be narrowed down to few postulates, if we consider every particle has a dedicated address in event horizon, every address has its own dedicated spacetime fabric, particles will have its projected shadows on other space time fabric and whenever there is interaction between true particle and other shadow particle, new address is assigned for both interacting particles and instantaneously all projected shadow becomes void. We perceive universe from collapse perspective only when particles collapse and new address are assigned, thus giving an illusion that we live in one universe but its result of interaction of multiple worlds. From new postulates we were able to explain how one of the entangled photons can decides other photons path in Wheeler's delayed choice experiment and thus helps us to removes problematic retro causality speculation to explain the phenomenon observed in this experiment.
\end{abstract}

Keywords: Quantum Mechanics Interpretation, Wave Function Collapse, Superposition,

Wheeler's Delayed Choice Experiment

\section{Introduction}

We will assume below 4 postulates to be valid for new interpretations and later each postulate will be proved valid based on existing principles and observation and help us to better understand these observation or principle from current interpretation.

1: Every particle has a dedicated address in event horizon.

2: Every address (irrespective of information stored or not) has its own dedicated spacetime fabric.

3: Particles will have their shadow (replicas) on the rest of the spacetime fabrics of other addresses.

4: Whenever a true particle interacts with a shadow particle, they get destroyed and create into new information (particles) with a new address and all old associated shadow particles disappear from other spacetime fabric and new shadow is projected on other spacetime fabrics.

Below we will try to prove why the above points are valid and thus helping us to understand the true mechanism behind working of Quantum mechanics.

\section{Every Particle Has Dedicated Address in Event Horizon}

\subsection{Bekenstein-Hawking Entropy}

Bekenstein-Hawking entropy is the amount of entropy a black hole must be assigned to comply with the laws of thermodynamics [1]. The black-hole entropy is equal to the ratio of the black-hole area to the square of the Planck length times a dimensionless constant of order unity. [2]

Figure 1 describes illustrates how information is stored in the event horizon of black hole, a quantized surface area holds the information about particles inside black hole and As per black hole cosmology model also called Schwarzschild cosmology or black hole cosmological is a cosmological model in which the observable universe is the interior of a black hole. Such models were originally proposed by theoretical physicist Raj Pathria, [3] and concurrently by mathematician I. J. Good. [4]. From 
Bekenstein-Hawking entropy and Schwarzschild cosmology we can conclude that every particle can have a dedicated address in the event horizon.

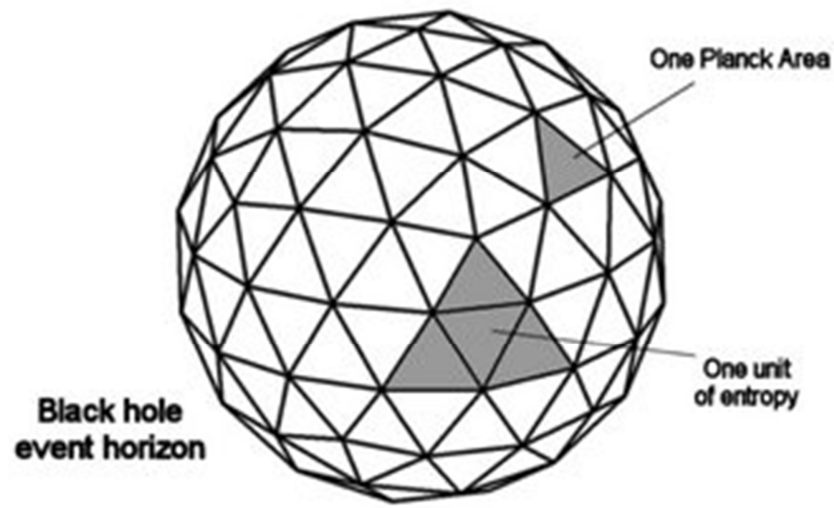

Figure 1. The Bekenstein-Hawking entropy is the entropy to be ascribed to any black hole: one quarter of its horizon area expressed in units of the Planck area.

\subsection{Entanglement}

When particles are entangled, they cannot be considered as individual particles, they are considered as inseparable entities. We need to consider other particles to describe one constituent fully. The state of an entangled particles is always expressible as a sum, or superposition, of products of states of each particles. When the entangled particles decohere (when a measurement is made or particle interacts with environment) entanglement is broken [5]. particle information is stored in the event horizon and maximally entangled particles are inseparable means these particles share the same address in the event horizon and as entangled particles can move in different directions means it should have its own spacetime fabric.

Whenever a measurement is made (by detector) on any one particle or when particle interacts with any other particle, there is interaction between the true particles address with the shadow particle address of detector (other particle) or vice versa, wherever particles interact they get assigned to a new address which is associated with the address where they interact, and thus now detector (or other particle) and particle becomes entangled (having address close to each other or same), other particle which was earlier entangled with old particle before measurement gets assigned new address based on location in same spacetime fabric where interaction occurred. Now as both old entangled particles are having new addresses these particles are not entangled any more, whenever a new address is assigned instantaneously all other spacetime fabric gets new shadow particles with new states. Thus, this interpretation even explains why particles are entangled and how measurement of one particle directly gives us information about other entangled particle (s) even if they are separated by huge distance and thus proves that every particle has dedicated address in event horizon

\section{Every Address (Irrespective of Information Stored or Not) Has Its Own Dedicated Spacetime Fabric}

Special Theory of Relativity

Minkowski diagrams are an extremely useful aid to visualizing how coordinates transform between different reference frames, it depends on velocity of the particles. 2 events may appear to be simultaneously to one observer but may not be simultaneous to another observer [6], this is explained well using Minkowski diagrams. For each observer (we will consider it as a particle for simplicity) it would appear they are still, and other particles are moving and other particles time (frequency) varies as per its velocity. Particles feel they are at rest and other observers are moving because their own address remains the same in its own spacetime fabric, but shadow particles projections keep changing.
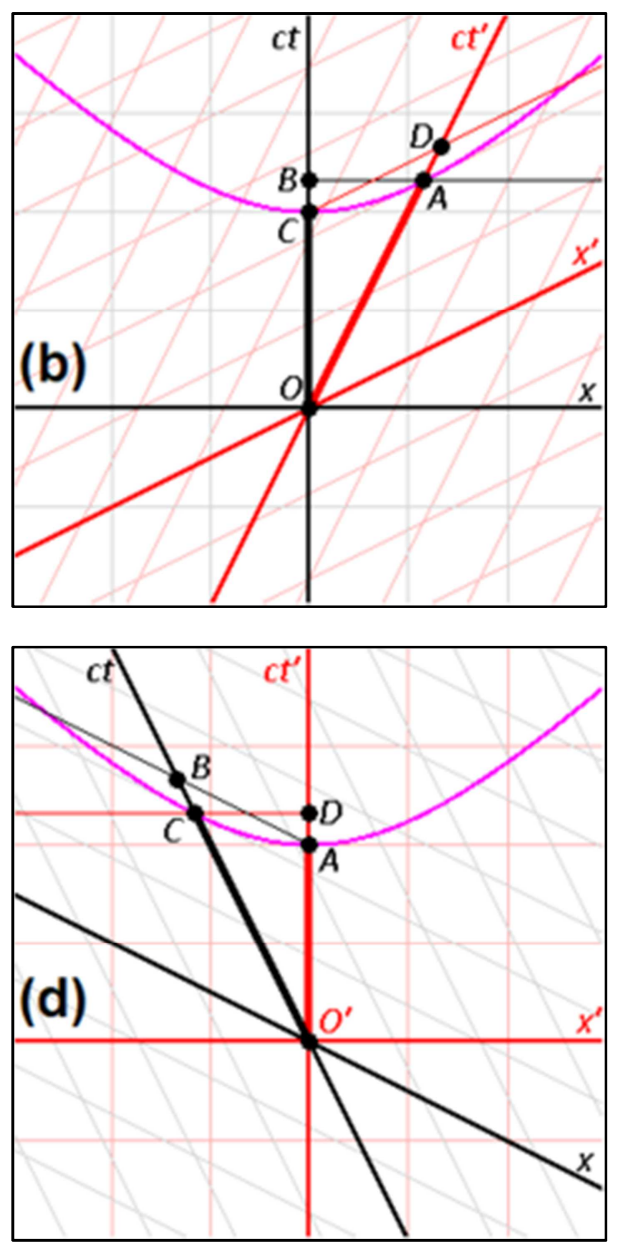

Figure 2. Minkowski diagrams showing measurements as seen from frame $S$ at rest"with un primed, rectangular axes, and frame $S^{\prime}$ thoving with $v>0$," coordinatized by primed, oblique axes, slanted to the right; the second picture shows frame $S^{\prime}$ ät rest"with primed, rectangular coordinates, and frame S thoving with $-v<0$,"with unprimed, oblique axes, slanted to the left.

In Figure in first figure (b) we see Minkowski diagrams showing particle $S$ frame of reference (at rest) with respect to $S$ ' frame of reference, which is shadow of $S$ ' particles on $S$ 
frame of reference and other figure (d) illustrates S' (at rest) with respect to $\mathrm{S}$ shadow particles frame of reference from above figures we can say that particles are not sharing same spacetime fabric, hence we can say each address will have different spacetime fabric and each particle having its own spacetime fabric also give provides reasoning behind lack of an absolute reference frame [7].

\section{True Particles Will Have Projections on the Other Spacetime Fabrics Addresses}

\section{Superposition}

Quantum Superposition states that, any two or more quantum states can be added together (superposed) and the result will be another valid quantum state; and conversely, that every quantum state can be represented as a sum of two or more other distinct states. Mathematically, it refers to a property of solutions to the Schrödinger equation [8].

Particles can exist in different states like they can be in different positions, have different energies or different speeds. Particles are thought of as existing across all the possible states at the same time. This means that a particle can be in two places or more places at once [9]. However, once a measurement of a particle is made (position or energy is known), the superposition is lost and now we have a particle in one known state.

A particle wavefunction is the sum of all wave functions for each address (true address and shadow address), whenever a shadow interacts with other shadows, they don't collapse the wave function but get super imposed which results in superposition. As true particle shadow is projected in all different fabrics of space for different addresses but each shadow can evolve to have slightly different states compared to true particle based on its own spacetime fabric constituents, hence the composite wave function of all wave functions of each address leads to superposition. Whenever an interaction occurs new address is assigned, and old shadow disappears hence we get information of only one state. Thus, superposition implies that true particles will have their shadow (replicas) on the rest of the spacetime fabrics for other addresses.

\section{Whenever a True Particle Interacts with a Shadow Particle, a New Address Is Assigned}

\subsection{Wave Function Collapse}

In quantum mechanics, wave function collapse occurs when a particle is in a superposition (multiple eigen states) reduces to a single eigenstate due to interaction with the external world [10]. As explained earlier a particle is in superposition due to its own interaction with shadow particles, when a measurement is made, measurement occurs in one of the spacetime fabric where a particle is having true address and detector has shadow address or vice versa, whenever a interaction happens between particle and detector, new address is assigned to both particle and detector which is copied to all other address instantaneously and thus we get snapshot of particle state at that time and thus giving us illusion of reading only one distinct state and as the address is modified all other states of old address becomes void.

Thus, the mechanism of assignment of new addresses gives the illusion of wave function collapse. Wave function collapse is not limited to only measurement it will occur whenever new address is assigned to particles with new information. Wave function collapse implies that whenever a true particle interacts with a shadow particle, they get destroyed and created into new information (particles) with a new address for each new particle and all associated shadow particles disappear from other spacetime fabric.

\subsection{Double Slit Experiment}

Consider double slit experiment where one electron at a time is bombarded to double slit, if we do not detect from which slit the electron has passed through we see interference pattern on screen [11], this is because electron on each address may pass through either first hole or second hole and due to interaction with all other shadow electrons wave function electron will form an interference pattern when electron wave function collapses on screen (only one position will captured as new address will be assigned during electron and screen particle interaction).

But when we detect an electron from which slit has passed through we only get to 2 distinct slits and no interference pattern, this is because when we detect electron from which slit it has passed though we modify the address of electron and that address becomes new source of new shadow electrons, as all shadow electrons are now passing through same slit there is no interference pattern (when new address is assigned all shadow electrons vanishes which has passed through another hole). Thus if we know which path the electron took in double slit experiment supports assumption that whenever a true particle interacts with a shadow particle, they get destroyed and create into new information (particles) with a new address for each new particle and all associated shadow particles disappear from other spacetime fabric.

\subsection{Wheeler's Delayed Choice Experiment}

The experimental setup of Wheeler's delayed choice experiment [12] is described in detail in Figure 3. An argon laser generates individual $351.1 \mathrm{~nm}$ photons that pass through a double-slit apparatus (vertical black line in the upper left corner of the diagram).

An individual photon goes through one (or both) of the two slits. In the illustration, the photon paths are color-coded as red or light blue lines to indicate which slit the photon came through (red indicates slit A, light blue indicates slit B). As per the experiment an entangled photon (signal photon) is 
detected first in D0 detector and another entangled photon (idler) is detected D1 or D2 or D3 or D4.

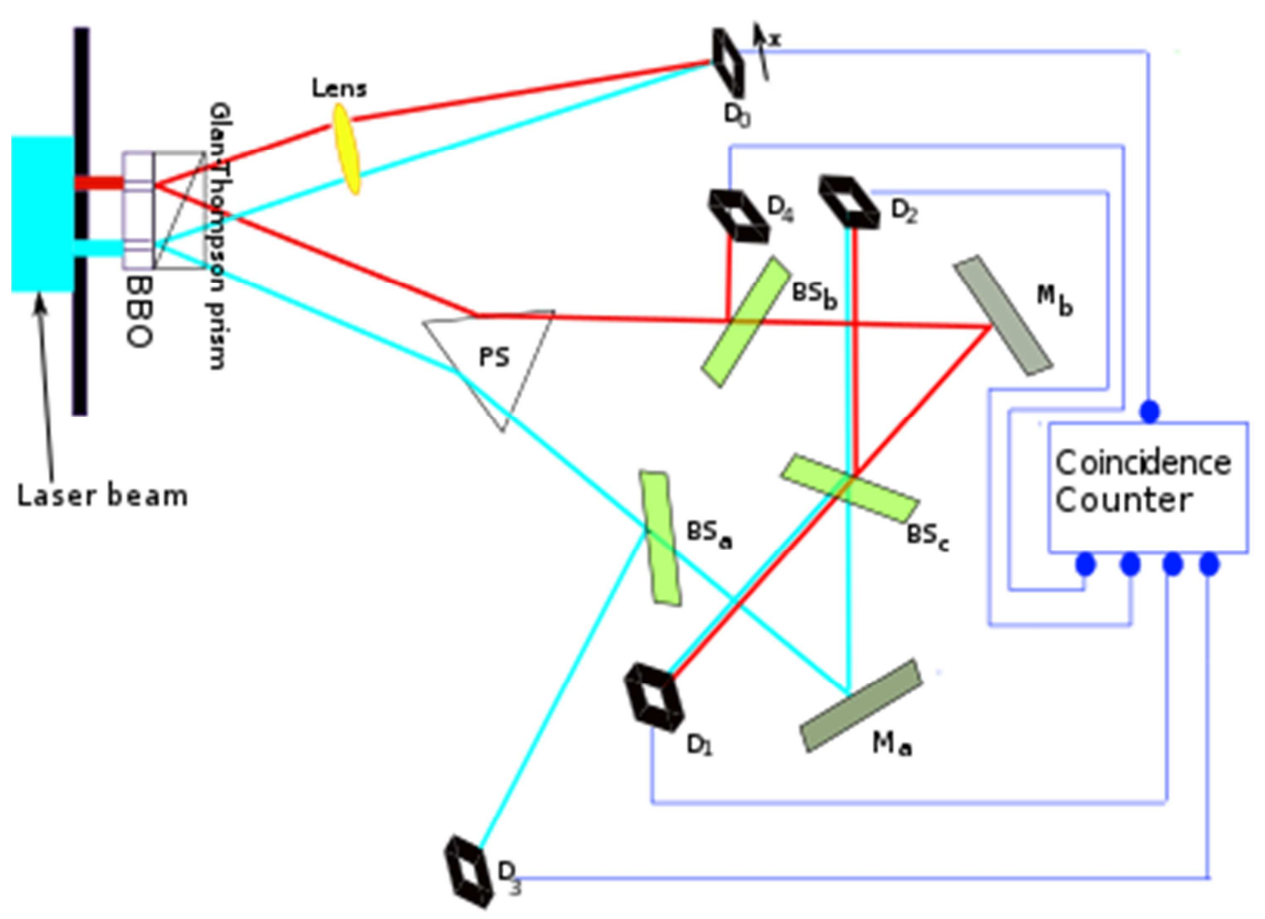

Figure 3. Setup of the delayed-choice quantum-eraser experiment of Kim et al. Detector $D_{0}$ is movable.

1) If an idler photon is recorded at detector D3, it can only have come from slit B.

2) If an idler photon is recorded at detector D4, it can only have come from slit A.

3) If an idler photon is detected at detector D1 or D2, it might have come from slit A or slit B.

4) The optical path length measured from slit to D1, D2, $\mathrm{D} 3$, and D4 is $2.5 \mathrm{~m}$ longer than the optical path length from slit to D0. This means that any information that one can learn from an idler photon must be approximately $8 \mathrm{~ns}$ later than what one can learn from its entangled signal photon.

5) When the experimenters looked at the signal photons whose entangled idlers were detected at D1 or D2, they detected interference patterns.

6) However, when they looked at the signal photons whose entangled idlers were detected at D3 or D4, they detected simple diffraction patterns with no interference.

As idler photon reaches D1, D2, D3 and D4 much later than signal photon reaches D0 there is speculation about retro causality because detectors D1, D2, D3 and D4 decides whether we would see interference pattern in D0 or not. Now as per current interpretation if photon is detected in D0, signal photon wave function collapses, and it is assigned new address, new address is also assigned to another entangled idler photon. When a signal photon is detected in a fabric of spacetime its partner will be having a path directing towards either to D1, D2, D3 or D4 on that same fabric of space. If an idler photon is D1 or D2 when signal photon was detected, shadow particles of idler photon from both paths would have already been superimposed and thus detector detects interference patterns. If during signal photon collapse the idler photon were headed towards D3 or D4, shadow photons interacting with the idler will be from the same path and won't create interference patterns. The collapse of the signal photon also modifies the address of the idler photon hence we detect the idler only in one detector and rest of all possibility vanishes automatically as the old address is void now. Detector D0 collapses idler photons to select a distinct path and detectors D1, D2, D3 and D4 detect this path later, which gives the illusion as if the idler photon decides if interference patterns can be detected in D0 or not. Thus, current interpretation removes retro causality from delayed choice experiment and explains whole mechanism based on assignment of new address for both entangled photon during detection of photon in detector D0.

\section{Schrödinger's Cat Paradox Explanation}

Schrödinger's cat: a cat, a flask of poison, and a radioactive source are placed in a sealed box. If an internal monitor (e.g. Geiger counter) detects radioactivity (i.e. a single atom decaying), the flask is shattered, releasing the poison, which kills the cat. [13] The Copenhagen interpretation of quantum mechanics implies that after a while, the cat is simultaneously alive and dead. Yet, when one looks in the box, one sees the cat either alive or dead, not both alive and dead. This poses the question of when exactly quantum superposition ends and reality collapses into one 
possibility or the other. The quantum-mechanical "Schrödinger's cat" paradox according to the Many-Worlds interpretation [14], every quantum event is a branch point; the cat is both alive and dead, even before the box is opened, but the "alive" and "dead" cats are in different branches of the universe, both of which are equally real, but which do not interact with each other.

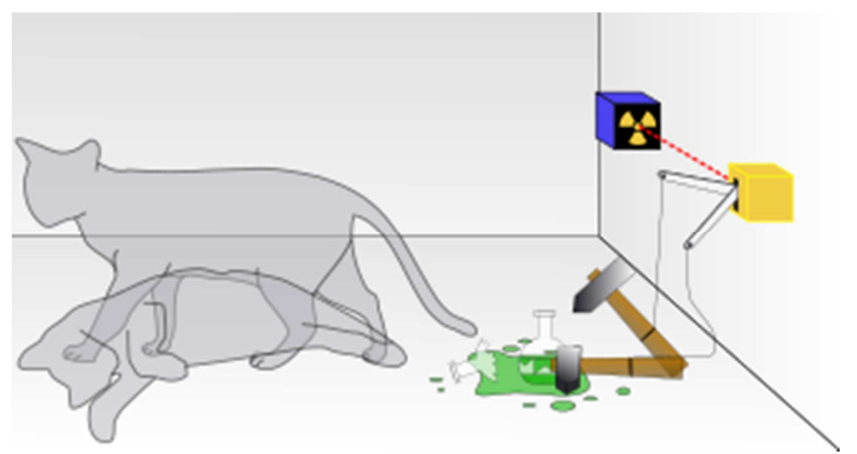

Figure 4. Schrödinger's cat: a cat, a flask of poison, and a radioactive source are placed in a sealed box.

But when you see series of events, there are multiple collapses (interaction) are happening even before box is opened, for example when radioactive decay occurs, when detector detects the decay, when hammer is released, flask is shattered, when poison interacts with cat, all this collapse will copy new states of each interaction in all worlds as per current implementation. Thus even before opening the box, cat state will already be copied in all world which would be either be dead or alive, just because we cannot measure the state we cannot know the current state, once we open the box there is another collapse which will be give us existing state of the cat before opening the box. Thus, this interpretation removes need of paradox of cat is alive and dead simultaneously in this experiment setup and need for new universe where cat is either be dead and alive simultaneously.

\section{Conclusion}

To understand quantum mechanics, we need to understand mechanism behind wave function collapse and entanglement. Current physics mathematically explains entanglement but fails to explain how the process works. "Emergent universe from many unreal world interpretation" gives mechanism behind wave function collapse and entanglement, by explaining how information is processed in holographic plane. This paper successfully explains weird phenomenon like particles in multiple location at once, Wheelers delayed choice experiment, wave particle duality using set of postulates without need of infinite branching universe used in many world interpretations. Universe which we experience, or measure is only snapshot of events and most of the underling reality cannot be observed directly but we can observe its impact on overall outcome.

\section{References}

[1] Jacob D. Bekenstein (2008) Bekenstein-Hawking entropy. Scholarpedia, 3 (10): 7375ei.

[2] D. Bekenstein Lett. Nuovo Cimento, 11 (1974), p. 467 arXiv: 1603.08674 [quant-ph]

[3] Pathria, R. K. (1972). "The Universe as a Black Hole". Nature. 240 (5379): 298-299. Bibcode: 1972Natur. 240.. 298P. doi: $10.1038 / 240298 \mathrm{a} 0$.

[4] Good, I. J. (July 1972). "Chinese universes". Physics Today. 25 (7): 15. Bibcode: 1972PhT....25g..15G. doi: 10.1063/1.3070923.

[5] Asher Peres, Quantum Theory, Concepts and Methods, Kluwer, 1993; ISBN 0-7923-2549-4 p. 115.

[6] Ashby, Neil (2003). Relativity in the Global Positioning System". Living Reviews in Relativity. 6 (1): 1. Bibcode: 2003LRR....6....1A. doi: 10.12942/lrr-2003-1. PMC 5253894. PMID 28163638.

[7] Staley, Richard (2009), "Albert Michelson, the Velocity of Light, and the Ether Drift", Einstein's generation. The origins of the relativity revolution, Chicago: University of Chicago Press, ISBN 0-226-77057-5.

[8] Zeilinger A (1999). "Experiment and the foundations of quantum physics". Rev. Mod. Phys. 71 (2): S288-S297. Bibcode: 1999RvMPS..71..288Z.

[9] Fein, Y. Y., Geyer, P., Zwick, P. et al. Quantum superposition of molecules beyond $25 \mathrm{kDa}$. Nat. Phys. 15, 1242-1245 (2019). https://doi.org/10.1038/s41567-019-0663-9.

[10] J. von Neumann (1932). Mathematische Grundlagen der Quantenmechanik (in German). Berlin: Springer.

[11] Eibenberger, Sandra; et al. (2013). "Matter-wave interference with particles selected from a molecular library with masses exceeding 10000 amu". Physical Chemistry Chemical Physics. 15 (35): 14696-14700. arXiv: 1310.8343. Bibcode: 2013PCCP...1514696E. doi: 10.1039/C3CP51500A. PMID 23900710.

[12] Kim, Yoon-Ho; R. Yu; S. P. Kulik; Y. H. Shih; Marlan Scully (2000). "A Delayed "Choice" Quantum Eraser". Physical Review Letters. 84 (1): 1-5. arXiv: quant-ph/9903047. Bibcode: 2000PhRvL..84...1K. doi: 10.1103/PhysRevLett.84.1. PMID 11015820.

[13] Schrödinger, Erwin (1935). "Die gegenwärtige Situation in der Quantenmechanik (The present situation in quantum mechanics)". Naturwissenschaften.

[14] Bryce S. DeWitt (1970). "Quantum mechanics and reality". Physics Today. 23 (9): 30. doi: 10.1063/1.3022331. See also Leslie E. Ballentine; Philip Pearle; Evan Harris Walker; Mendel Sachs; Toyoki Koga; Joseph Gerver; Bryce DeWitt (1971). "Quantum-mechanics debate". Physics Today. 24 (4): 36. doi: $10.1063 / 1.3022676$. 